\title{
A survival analysis of invasive fungal diseases in children with
}

\section{cancer}

\author{
Letícia Marques $^{1}$, Orlei Araujo ${ }^{2}$, Adriana Silva ${ }^{3}$, Dafne Bourguignon da Silva ${ }^{4}$, and \\ Fabianne Carlesse ${ }^{5}$ \\ ${ }^{1}$ Infection Control Committee, Institute of Pediatric Oncology - Support Group for \\ Adolescents and Children with Cancer/Federal University of Sao Paulo \\ (IOP-GRAACC/UNIFESP), Sao Paulo, Brazil. \\ ${ }^{2}$ UNIFESP \\ ${ }^{3}$ Infection Control Committee, Institute of Pediatric Oncology - Support Group for \\ Adolescents and Children with Cancer/Federal University of Sao Paulo \\ (IOP-GRAACC/UNIFESP), Sao Paulo, Brazil \\ ${ }^{4}$ Intensive Care Unit, Institute of Pediatric Oncology - Support Group for Adolescents and \\ Children with Cancer/Federal University of Sao Paulo (IOP-GRAACC/UNIFESP), Sao \\ Paulo, Brazil. \\ ${ }^{5}$ Universidade Federal de São Paulo
}

October 28, 2020

\begin{abstract}
Background: Invasive fungal diseases (IFD) are important causes of mortality in children with cancer. We aimed to determine the extent of survival in patients treated for IFD in the last nine years at our center. Procedure: a retrospective cohort of patients treated from January 1, 2011 to December 31, 2019. Weibull distribution was used to parameterize hazard ratios and accelerated failure time models, for the outcome "death attributed to IFD". Results: We analyzed 152 patients with IFD (133 proven and 19 probable), with median age of 97 months. The most frequent diagnoses were leukemia (39, 25.7\%) and central nervous system tumors $(36,23.7 \%)$. Thirty-seven patients received prophylaxis with fluconazole $(24.3 \%)$. There were 133 fungi isolates, and most frequent were Candida species in blood $(84,55.2 \%)$. Forty-three deaths were attributed to IFD (28.3\%). Survival probabilities were lower for pulmonary IFD $(46.9 \%, \mathrm{p}=0.0017)$, leukemia $(62.5 \%, \mathrm{p}=0.004)$, and neutropenia $<500$ cells $/ \mathrm{mm} 3$ (55.4\%, $\mathrm{p}<0.0001)$. For Candida fungemia, survival probabilities were $76.6 \%(\mathrm{p}=0.043)$. In Weibull models, diagnosis of leukemia shortened survival times by a factor of 0.006 , relapse of disease by 0.05 , lymphoma by 0.04 , pulmonary IFD by 0.04 , and neutropenia by 0.015 . Hematopoietic stem cell transplantation did not affect the survival times, as well as prophylaxis with fluconazole. Conclusions: Host factors, like neutropenia, relapse of disease and hematologic malignancies, are determinant in the survival times of children with IFD, as well as pulmonary involvement. Fluconazole prophylaxis and HSCT do not affect the hazards of death.
\end{abstract}

\section{Introduction}

Invasive fungal diseases (IFD) are important causes of morbidity and mortality in immunocompromised children and adolescents with cancer. Factors that have been linked to the development of IFD in children and adults are the underlying malignancy (mainly acute leukemia), presence of profound and long-lasting neutropenia, high intensity of the chemotherapeutic regimen, hematopoietic stem cell transplantation, and previous antibiotic therapy. ${ }^{1}$ Candida species (spp.) are commonly found in the gastrointestinal tract, and 
may cause invasive infections in the immunocompromised host when mucosal barrier is disrupted, or normal gastrointestinal flora is modified by antimicrobial treatment. ${ }^{2}$ Filamentous fungi, including the Zygomycetes class, Fusarium and Aspergillusspecies have also become epidemiologically relevant in this population. ${ }^{3}$

The main objective of this study was to determine the extent of survival of children and adolescents with cancer, treated for IFD in the last nine years, at a referral center for the treatment of childhood cancer in Brazil. In addition, we aimed to analyze which factors could be determinant to shorten survival.

\section{Methods}

We analyzed a retrospective cohort of patients who were treated at our institution, the Institute of Pediatric Oncology - Support Group for Adolescents and Children with Cancer/Federal University of Sao Paulo (IOPGRAACC/UNIFESP), from January 1, 2011 to December 31, 2019. Patients aged 0 to 18 years, diagnosed with cancer, submitted or not to hematopoietic stem cell transplantation, were included. We analyzed only patients who had proven or probable invasive fungal infection, as defined by the consensus from the European Organization for Research and Treatment of Cancer and the Mycoses Study Group Education and Research Consortium. ${ }^{4}$ The study was approved by the Ethics and Research Committee of the Federal University of São Paulo.

Variables collected included the subject's demographic characteristics, underlying disease, type and site of IFD, antifungals drugs, and outcomes. Death was considered as resulting from the fungal infection when it occurred because of clinical deterioration with evidence of a proven or probable IFD.

For survival analyses, we used the Weibull distribution to parameterize hazard ratios and accelerated failure time models (AFT). An AFT model assumes that the effect of a covariate is to accelerate or decelerate the life course of a disease by some constant ${ }^{5}$ Conditions like the diagnoses, relapse of disease, hematopoietic stem cell transplant, use of antifungal drugs at the time of diagnosis of IFD, presence of neutropenia, age, and site of infection were tested in models for the outcome "death caused by IFD". Kaplan Meier curves were also made for this outcome, with comparisons using the Log Rank test (Mantel-Cox). All the statistical tests were performed with R software, version 4.0.2 (The R Foundation for Statistical Computing, 2020).

\section{Results}

We analyzed 152 IFD (133 proven and 19 probable). All probable IFD were pulmonary aspergillosis, with high-resolution computerized tomography scans presenting dense, well-circumscribed lesions with a halo sign, air crescent sign, cavities or segmental or lobar consolidation, with galactomannan antigen detected in plasma. Table 1 depicts the demographics and other characteristics of patients. There were 133 fungal isolates in 133 patients (table 2).

[Table 1]

[Table 2]

There were 43 deaths attributed to IFD (28.3\%), and survival probabilities were lower for pulmonary IFD $(46.9 \%, \log$ rank test $\mathrm{p}=0.0017)$, for the diagnosis of leukemia $(62.5 \%, \mathrm{p}=0.004)$, and neutropenia $<500$ cells $/ \mathrm{mm} 3(55.4 \%, \mathrm{p}<0.0001)$. For Candida fungemia, survival probabilities were higher $(76.6 \%, \mathrm{p}=0.043)$. Figure 1 depicts the Kaplan Meier survival curves for these conditions. Table 3 shows the Weibull hazard ratios and accelerated failure time models for the event "death caused by IFD".

[Figure 1]

[Table 3]

Most of the cases of leukemia $(51 / 56,91 \%)$ were in children older than 4 years; about $72 \%$ of the patients were older than 4 years, but out of the 43 deaths, 38 (88\%) were in this age group. Non-Candida and nonCryptococcus yeasts caused fungemia in 16 patients, with three deaths $(18.7 \%)$. In four patients, there were pulmonary lesions accompanying Candida fungemia, and Cryptococcus was isolated in lung biopsy in one case; all other cases of fungal pneumonia were associated with filamentous fungi. Pulmonary involvement 
could be correlated in chi-square tests with the diagnosis of leukemia $(18 / 56$ cases, $32 \%, \mathrm{p}=0.01)$, relapse of disease $(17 / 41$ cases, $41 \%, \mathrm{p}=0.0002)$, and neutropenia $(23 / 74$ cases, $31 \%, \mathrm{p}=0.0002)$.

In the HSCT patients, there were eight deaths (25.8\%). Fungemia was diagnosed in 16 patients (51.6\%) and pulmonary fungal infections in $12(38.7 \%)$.

\section{Discussion}

In this work, we showed that host factors, particularly neutropenia and hematologic malignancies, are determinant in the survival times of children affected with IFD. The accelerated failure time models estimate the effects of the variables on acceleration or deceleration on the survival times: so, we demonstrated that the children with leukemia, besides having multiplied the hazard for death due to IFD by 2.5 , also could have their survival time shortened by a factor of 0.006 . Among the several reasons why patients with leukemia are at risk of dying from IFD, one of the most important is neutropenia, which was prolonged in this cohort, and was a determinant factor for shortening survival in our analysis. Many patients with leukemia have severe, prolonged neutropenia caused by the disease itself or chemotherapy, and mucosal lesions that can predispose to infection. ${ }^{6}$ We analyzed few cases of lymphoma, but this diagnosis was also a factor to shorten survival in patients with IFD.

The overall mortality reported here, around $28 \%$, is identical to that reported by Castagnola et al, in $2006 .^{7}$ After 14 years, we are using the same therapeutic options, and little progress has been made in the development of new antifungal agents. ${ }^{8}$ Another point of similarity with the study of Castagnola et al. was the lower mortality in patients with fungemia, compared to other IFD. ${ }^{7}$ The high mortality observed in pulmonary infections can be explained by several factors: as noted, pulmonary involvement was correlated with the conditions that most favored mortality from IFD, such as the diagnosis of leukemia, cancer recurrences and neutropenia. The most life-threatening form of aspergillus infection, pulmonary angioinvasive aspergillosis, occurs in immunosuppressed or neutropenic patients: the lung shows a necrotizing pneumonia with areas of hemorrhage and acute and granulomatous inflammation. ${ }^{9}$ Over the last years, there has been a reduction of mortality due to invasive aspergillosis in those patients with myeloid leukemia, in whom remission of malignancy and effective antifungal treatments can be attained, but mortality can grew up to $54 \%$ in relapsed/refractory disease, reflecting the compromised immune status of these patients. ${ }^{10}$ Pulmonary zygomycoses andFusarium spp. pneumonia also have high fatality rates. ${ }^{11,12}$

The use of empirical antifungal drugs at the time of diagnosis of IFD affected the survival negatively, but the prophylaxis with fluconazole did not. This finding probably means that the children who received empirical treatment were at higher risk of severe infections, or presented a clinical deterioration, requiring the coverage with antifungals. The risk of death was also 3.8 -fold higher in patients already receiving antifungals at the time of diagnosis of IFD in the study of Castagnola et al., but these authors analyzed prophylaxis and empiric use as a single variable. ${ }^{7}$

Candidemia is now a frequent cause of bloodstream infection, due to the widespread use of central venous catheters, parenteral nutrition, broad-spectrum antibiotics, and immunosuppressive therapy. Rare opportunistic yeasts are emerging as cause of IFD. ${ }^{13}$ We observed 16 episodes of fungemia caused by these rare yeasts, but mortality was similar to observed in candidemia.

Allogeneic unrelated HSCT or mismatched donor HSCT put the patient in the highest risk group for IFD. ${ }^{14}$ Compared with allogeneic HSCT, autologous HSCT is associated with a lower risk of invasive aspergillosis because the period of pre-engraftment neutropenia is shorter and the possibility of graft-versus-host disease is absent. ${ }^{15}$ Although the mortality of our HSCT patients with IFD was still high (25.8\%), and the fact that most were allogeneic, the transplantation was not a factor for shortening survival. One explanation is that HSCT is the best chance of cure for diseases like myeloid leukemia, ${ }^{16}$ and most of the patients will recover from neutropenia.

The most obvious weakness of this study, common to several others of its kind, is the absence of sensitivity tests to antifungals, not routinely performed in our institution. The results are also limited to patients treated 
in a single center, and may not reflect practices from other parts of our country or the world.

\section{Conclusions}

Host factors, like neutropenia, relapse of disease, and hematologic malignancies, are determinant in the survival times of children affected with IFD. Pulmonary fungal infections, most caused by filamentous fungi, are also responsible for shortening survival times in these patients. Fungemia, most caused by Candida species, seems to lead to a better survival. Fluconazole prophylaxis does not appear to affect the hazards of death from IFD.

1. Mor M, Gilad G, Kornreich L, Fisher S, Yaniv I, Levy I. Invasive fungal infections in pediatric oncology. Pediatr Blood Cancer. 2011;56(7):1092-1097. doi:10.1002/pbc.23005

2. Gokcebay DG, Yarali N, Isik P, et al. Candida Associated Bloodstream Infections in Pediatric Hematology Patients: A Single Center Experience. Mediterr J Hematol Infect Dis. 2016;8(1):e2016018. doi:10.4084/MJHID.2016.018

3. Morris SK, Allen UD, Gupta S, Richardson SE. Breakthrough filamentous fungal infections in pediatric hematopoetic stem cell transplant and oncology patients receiving caspofungin. Can J Infect Dis Med Microbiol. 2012;23(4):179-182. doi:10.1155/2012/957973

4. Donnelly JP, Chen SC, Kauffman CA, Steinbach WJ, Baddley JW, Verweij PE, et al. Revision and Update of the Consensus Definitions of Invasive Fungal Disease From the European Organization for Research and Treatment of Cancer and the Mycoses Study Group Education and Research Consortium. Clin Infect Dis. 2020;71(6):1367-1376. doi: 10.1093/cid/ciz1008. PMID: 31802125

5. Karimi A, Delpisheh A, Sayehmiri K. Application of accelerated failure time models for breast cancer patients' survival in Kurdistan Province of Iran. J Cancer Res Ther. 2016;12(3):1184-1188. doi: 10.4103/0973-1482.168966. PMID: 28054533

6. Bhatt VR, Viola GM, Ferrajoli A. Invasive fungal infections in acute leukemia. Ther Adv Hematol. 2011;2(4):231-247. doi:10.1177/2040620711410098

7. Castagnola E, Cesaro S, Giacchino M, et al. Fungal infections in children with cancer: a prospective, multicenter surveillance study. Pediatr Infect Dis J. 2006;25(7):634-639. doi:10.1097/01.inf.0000220256.69385.2e

8. Scorzoni L, de Paula E Silva AC, Marcos CM, et al. Antifungal Therapy: New Advances in the Understanding and Treatment of Mycosis. Front Microbiol. 2017;8:36. doi:10.3389/fmicb.2017.00036

9. Kradin RL, Mark EJ. The pathology of pulmonary disorders due to Aspergillus spp. Arch Pathol Lab Med. 2008 Apr;132(4):606-14. doi: 10.1043/1543-2165(2008)132[606:TPOPDD]2.0.CO;2. PMID: 18384212

10. Dragonetti G, Criscuolo M, Fianchi L, Pagano L. Invasive aspergillosis in acute myeloid leukemia: Are we making progress in reducing mortality? Med Mycol. 2017 Jan 1;55(1):82-86. doi: 10.1093/mmy/myw114. Epub 2016 Dec 2. PMID: 27915304

11. Petrikkos G, Drogari-Apiranthitou M. Zygomycosis in Immunocompromised non-Haematological Patients. Mediterr J Hematol Infect Dis. 2011;3(1):e2011012. doi:10.4084/MJHID.2011.012

12. Nucci M, Anaissie E. Fusarium infections in immunocompromised patients. Clin Microbiol Rev. 2007;20(4):695-704. doi:10.1128/CMR.00014-07

13. Fernández-Ruiz M, Guinea J, Puig-Asensio M, Zaragoza Ó, Almirante B, Cuenca-Estrella M, et al; CANDIPOP Project; GEIH-GEMICOMED (SEIMC) and REIPI. Fungemia due to rare opportunistic yeasts: data from a population-based surveillance in Spain. Med Mycol. 2017;55(2):125-136. doi: $10.1093 / \mathrm{mmy} / \mathrm{myw} 055$

14. Bhatt VR, Viola GM, Ferrajoli A. Invasive fungal infections in acute leukemia. Therapeutic Advances in Hematology. 2011:231-247. doi:10.1177/2040620711410098

15. Wiederhold NP, Lewis RE, Kontoyiannis DP. Invasive aspergillosis in patients with hematologic malignancies. Pharmacotherapy. 2003;23(12):1592-610. doi: 10.1592/phco.23.15.1592.31965. PMID: 14695039

16. Medinger M, Lengerke C, Passweg J. Novel therapeutic options in Acute Myeloid Leukemia. Leuk Res Rep. 2016;6:39-49. doi:10.1016/j.lrr.2016.09.001 
Legend of figure 1:

Figure 1. Kaplan Meier survival curves in IFI, for the diagnoses of leukemia, presence of neutropenia, pulmonary fungal infections and fungemia by Candida species. P-values refer to the log rank test.

\section{Hosted file}

Table 1.pdf available at https://authorea.com/users/370832/articles/489357-a-survivalanalysis-of-invasive-fungal-diseases-in-children-with-cancer

\section{Hosted file}

Table 2.pdf available at https://authorea.com/users/370832/articles/489357-a-survivalanalysis-of-invasive-fungal-diseases-in-children-with-cancer

\section{Hosted file}

Table 3.pdf available at https://authorea.com/users/370832/articles/489357-a-survivalanalysis-of-invasive-fungal-diseases-in-children-with-cancer
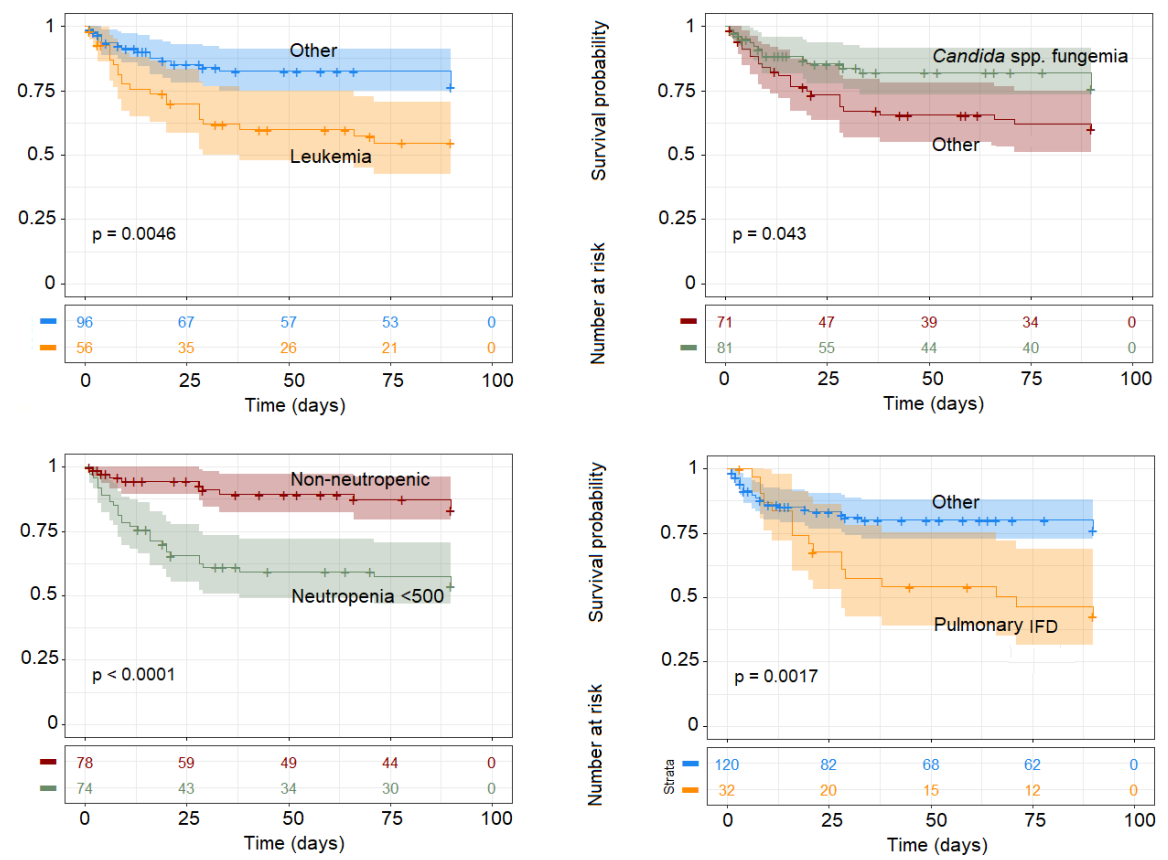